Revue d'histoire des chemins de fer

\section{Revue d'histoire des chemins de fer}

26 | 2003

Normalisation ferroviaire, cultures de réseaux -

L'Europe des chemins de fer, 1878-2000

\title{
Diversité ou unité des exploitations ferroviaires : un antagonisme permanent?
}

\section{Georges Ribeill}

\section{OpenEdition}

\section{Journals}

Édition électronique

URL : https://journals.openedition.org/rhcf/1937

DOI : 10.4000/rhcf.1937

\section{Éditeur}

Rails \& histoire

\section{Édition imprimée}

Date de publication : 1 février 2003

Pagination : 16-42

ISBN : 0996-9403

ISSN : 0996-9403

\section{Référence électronique}

Georges Ribeill, « Diversité ou unité des exploitations ferroviaires : un antagonisme permanent? 》, Revue d'histoire des chemins de fer [En ligne], 26 | 2003, mis en ligne le 19 janvier 2015, consulté le 22 avril 2022. URL : http://journals.openedition.org/rhcf/1937 ; DOI : https://doi.org/10.4000/rhcf.1937 


\section{Diversité ou unité des exploitations ferroviaires : un antagonisme permanent ?}

\section{Des facteurs permanents favorables à la normalisation}

Alors que l'exploitant ferroviaire est naturellement attaché à sa liberté de conception et d'innovation, c'est plutôt du côté des administrations de tutelle et de contrôle que, pour diverses raisons, voient le jour des préoccupations normalisatrices, traduites en contraintes imposées aux exploitants relevant de leur autorité.

\section{Le rôle normatif minimum d'une forte tutelle}

A première vue, les réseaux français d'intérêt général ont adopté et pratiqué depuis leurs origines des normes fondamentales. La plus manifeste concerne l'écartement de la voie : depuis la concession du Paris-Saint-Germain (1835) où il est fixé à 1,44 m entre les bords intérieurs des rails, il est imposé dans tous les cahiers des charges ultérieurs ${ }^{1}$ et fixé précisément à $1,435 \mathrm{~m}$.

Par le biais de ces cahiers des charges imposés aux exploitants par la tutelle du ministère des Travaux publics, toute une série d'autres normes implicites, minimales, régissent l'infrastructure et l'exploitation technique, dont les justifications relèvent principalement de la sécurité. Bien plus que la loi du 11 juin 1842 qui définit le régime de référence des chemins de fer «à la française » (régime de concession monopolistique, sous forte tutelle étatique), la loi de police des chemins de fer du 15 juillet 1845 et l'ordonnance du 15 novembre 1846 qui en précise les modalités d'application imposent de la même manière à toutes les compagnies des contraintes et sujétions tant techniques que commerciales, visant la conception comme l'exploitation de leurs chemins de fer. De quoi irriter durablement les compagnies qui objectent par exemple que l'espacement et la largeur réglementaires des banquettes et sièges qui leur sont imposés en fonction des trois classes de confort offertes ne relèvent nullement de la sécurité de l'exploitation...

1- A l'exception toutefois de la ligne de Paris à Sceaux (1844), où l'on autorise l'ingénieur Arnoux à tester sur une ligne à très fortes courbures un système de wagons articulés dont le mouvement sera facilité de surcroît par un écartement des rails élargi à $1,75 \mathrm{~m}$. 
Ces normes premières se multiplient vite. Les ingénieurs fonctionnaires chargés du contrôle technique de l'exploitation n'auront de cesse, à l'issue de chaque catastrophe ferroviaire, d'ériger en nouvelles normes et prescriptions réglementaires des techniques et procédures novatrices qui leur semblent constituer des moyens appropriés de prévenir le renouvellement de ces catastrophes.

Par ailleurs, la promotion rapide des chemins de fer en service public de transport, en garantissant aux voyageurs une égalité de traitement commercial ou l'accès au choix à trois classes de places, conforte aussi le rôle normatif des pouvoirs de tutelle et de contrôle sur les compagnies dans le domaine de la définition de l'offre ferroviaire.

Enfin, bien que plus discrètement, les autorités militaires vont continûment peser sur le domaine ferroviaire : jusqu'en 1939, en bloquant l'électrification des lignes proches de la frontière nord-est, elles érigent bien en norme restrictive sur une large partie du territoire français la traction à vapeur ou autonome. Et pour ces mêmes lignes du Nord-Est, la prise en considération de l'intérêt stratégique militaire imposa lors de leur conception aux tracés en vallée de suivre la rive occidentale, c'est-à-dire de protéger la voie ferrée des éventuels assauts ennemis par la voie d'eau contiguë, quel que soit l'éventuel surcoût de ce choix ${ }^{2}$.

\section{Des facilités de circulation ferroviaire d'un réseau à l'autre}

Alors que les cahiers des charges prévoient, moyennant péage, la libre circulation des trains d'un réseau d'intérêt général à l'autre dès lors que leurs lignes sont connexes ${ }^{3}$, hormis l'écartement standard cité plus haut, rien n'est imposé d'une concession à l'autre qui viserait à faciliter les circulations et échanges de matériel moteur et roulant entre réseaux ${ }^{4}$ : les compagnies françaises ont joué plutôt la règle tacite du «chacun

2- La Géographie des chemins de fer français de H. Lartilleux (Librairie Chaix) permet très commodément (cartes p. 34 et 134 de l'édition de 1951) de vérifier cette règle, que nous avons vue explicitement confirmée dans son application au tracé de la ligne de Lyon arrêté en 1845 : les autorités militaires imposèrent aux ingénieurs des Ponts et Chaussées de ne pas faire franchir l'Yonne par le chemin de fer à hauteur de Pont-sur-Yonne, où le tracé sur la rive gauche venait buter sur une imposante colline ; il fallut y percer donc une importante et très coûteuse tranchée. 3- Voir G. Ribeill, «Le principe du libre parcours sur les premiers chemins de fer concédés. Fondements théoriques et obstacles pratiques », in « Les chemins de fer en temps de concurrence : choix du XIX siècle et débats actuels ", actes du $\sigma^{\text {e }}$ colloque de l'AHICF, Revue d'histoire des chemins de fer, $\mathrm{n}^{\circ}$ 16-17 (printempsautomne 1997), p. 29 et sq.

4- Ce que le terme récent et commode d'interopérabilité érige en enjeu contemporain essentiel des transports ferroviaires internationaux. 
chez soi ", au prix de transbordements coûteux des marchandises et de correspondances pas toujours bien assurées entre trains de voyageurs dans les gares de jonction.

En revanche, poussées par le principe économique des rendements croissants qui régit leur compte d'exploitation ${ }^{5}$, favorables à des transports massifs et à grande distance, d'idéologie libre-échangiste plutôt que protectionniste, les compagnies françaises ont cherché à faciliter les trafics internationaux de part et d'autre des frontières politiques, y compris les trafics de pur transit. Les gouvernements de l'Europe continentale ont cherché aussi, par le relais d'accords diplomatiques, à faciliter les exportations, au prix d'importations réciproques. Le rail étant alors le mode le plus approprié, du coup, il est naturel d'observer la multiplication précoce des institutions internationales à compétence ferroviaire (administrations et/ou exploitants), vouées à faciliter ces échanges commerciaux.

C'est à Berne, en 1882, qu'une conférence rapprochant divers réseaux et Etats se propose de définir un règlement commun, dit Unité technique des chemins de fer (UT), fixant les conditions techniques de sécurité auxquelles doivent répondre les voies ferrées et les véhicules pour assurer le transit international ${ }^{6}$. Malgré leur développement (voir Annexe 1), la longueur des procédures aboutissant à des accords internationaux sera d'autant plus grande que les membres participant seront nombreux à défendre leurs particularismes, à vouloir les imposer en normes internationales ${ }^{7}$. D'un autre côté, les questions traitées périodiquement par exemple depuis sa création en 1885 au Congrès international des chemins de fer et intéressant les thèmes de la normalisation et de l'interopérabilité s'avèrent plutôt rares et de moins en moins fréquentes (voir Annexe 2).

Bien différent d'une France unifiée et jacobine, le contexte politique du XIX siècle qui encadre le développement ferroviaire de tout petits

5- Schématiquement, le coût total d'acheminement de la $n+1^{\mathrm{e}}$ unité de trafic (voyageur ou tonne supplémentaire) est moins élevé que celui de la $\mathrm{n}^{\mathrm{e}}$.

6- La fameuse clef de Berne, ce « sésame » de la plupart des serrures du matériel ferroviaire dont disposent tous les cheminots roulants d'Europe, symbolise parfaitement cette ouverture et cette interpénétration facilitée entre réseaux.

7- Par exemple, la révision de la Rédaction 1913 du règlement de l'Unité technique, suggérée en 1924 par l'Union internationale des chemins de fer (UIC), conduit le gouvernement suisse, gérant de l'Unité technique, à formuler un premier projet approuvé en 1929 par l'UIC, c'est-à-dire les réseaux, mais critiqué par certains États ; d'où un second projet adopté par l'UIC en 1935 et seulement en 1938 par les gouvernements (voir « Unité technique des chemins de fer », Revne générale des chemins de fer, mai-juin 1940, p. 164). 
États allemands et de certains États périphériques y a motivé très tôt, dès 1846, la fondation d'une association internationale vouée à assurer l'interopérabilité entre réseaux membres : le Verein. Depuis 1856, une Convention technique pour la construction et l'exploitation des chemins de fer définit les règles imposées à tout réseau adhérent et, à partir de 1868, un règlement organise l'échange de matériel. En revanche, toute liberté est laissée aux réseaux en matière tarifaire ${ }^{8}$. Significative est la discrétion des articles qu'en France la Revue générale des chemins de ferconsacre à relater les activités du Verein; et dans la première notice qu'elle lui consacre?, l'auteur, un inspecteur attaché aux lignes du Nord-Belge ${ }^{10}$, s'étonne de la coexistence paradoxale de « deux conditions qui paraissent appelées bien plus à s'exclure qu'à se combiner» :

«Le réseau des voies ferrées est très morcelé, et pourtant le service n'y subit pas les graves inconvénients qui résultent toujours d'administrations multiples. Il porte, au contraire, au point de vue technique, la trace de l'unité dans les principes essentiels tant de l'exploitation que de la construction, et une uniformité accentuée se manifeste dans les applications de ces principes. »

\section{L'intérêt économique des constructeurs de matériel ferroviaire}

En 1899, dans un contexte de crise des commandes de la part des compagnies et de sévère concurrence étrangère, allemande notamment $^{11}$, les constructeurs français de matériel roulant s'organisent enfin en chambre syndicale. Leur manque de compétitivité, expliquent-ils, provient de la nature des commandes des compagnies françaises, réduites à de courtes séries de matériel de types toujours changeants, donc grevés pour ces deux raisons d'un important surcoût. Leur préoccupation sera bien entendue par le ministre des Travaux publics Pierre

8- Le mode de décision accorde une voix à tout réseau adhérent de moins de $100 \mathrm{~km}$ et un nombre dégressif de voix aux autres réseaux (de 100 à $250 \mathrm{~km}$, 2 voix ; de 1500 à $2000 \mathrm{~km}, 8$ voix...). En 1879, le Verein regroupe 110 réseaux d'une longueur totale de 53400 kilomètres. L'Allemagne domine avec $58 \%$ des lignes et $55 \%$ des voix, devant l'Autriche-Hongrie (35\% des lignes et autant des voix) et divers autres réseaux belges, luxembourgeois, hollandais, polonais et roumains ( $8 \%$ des lignes et $10 \%$ des voix). Alors que l'unanimité est de règle s'agissant de modifications dans les statuts, $9 / 10^{\circ}$ des voix suffisent pour l'adoption de règles opérationnelles communes.

9- M. Philippe, "Notice sur l'Union (Verein) des chemins de fer allemands », Revue générale des chemins de fer, avril 1879.

10- Lignes dépendant de la Compagnie française du Nord.

11- Thème central de l'ouvrage d'Allan Mitchell, The Great Train Race. Railways and the Franco-German Rivalry, New York-Oxford, Berghahn Books, 2000. 
Baudin qui, disposant d'un droit de contrôle sur leur matériel, avise par une circulaire du 18 août 1900 les administrateurs des compagnies qu'ils ne seront plus désormais aussi libres qu'auparavant de choisir leurs nouveaux types de matériel ${ }^{12}$ :

«Mon attention a été appelée sur les inconvénients que présente l'extrême diversité des types en usage sur les chemins de fer français pour le matériel roulant et principalement pour les machines locomotives. Non seulement chaque Compagnie a ses modèles spéciaux, mais, dans l'intérieur du même réseau, il est rare qu'une commande nouvelle n'apporte pas aux types précédents des modifications de détail qui obligent chaque fois les services de traction à des études fort longues et les constructeurs à l'établissement de dessins d'exécution et de modèles fort coûteux. [...].

Pour remédier à cet état de choses, je me propose de prescrire aux ingénieurs en chef du contrôle technique d'examiner, à l'occasion de chacun des projets de matériel roulant soumis à mon approbation, s'il n'existe pas déjà de type analogue ayant fait ses preuves et d'exiger, à l'appui des modifications proposées à ces types, des justifications qui en démontrent la nécessité.

Je vous prie de votre côté de bien vouloir tenir compte, lors de la préparation des projets, des considérations qui précèdent [...]. »

Par un arrêté du 4 mai 1901, Baudin crée une commission « chargée de codifier les cahiers des charges en vigueur pour la fourniture des métaux et des matières entrant dans la fourniture du matériel roulant des chemins de fer et d'unifier les types de matériel ». Les instructions communiquées en date du 16 mai $1901^{13}$ à son président, le directeur des Chemins de fer Jules Lax, sont très précises quant aux résultats recherchés :

«La première mesure à prendre pour amener à la réduction des prix doit être d'établir plus d'uniformité dans les éléments des principaux types de matériel et dans les cahiers des charges relatifs aux fournitures. Ce sera là un des résultats les plus appréciables de l'étude à laquelle vous allez vous livrer. »

Plus précisément, la mission devait :

12- Archives de la Fédération des industries ferroviaires, Paris.

13- J.O., 16 mai 1901, p. 3114. 
« $2^{\circ} /$ proposer, principalement pour les machines locomotives, les données caractéristiques d'un certain nombre de types, auxquels les compagnies devront se conformer, pendant une période à déterminer, pour leurs demandes d'autorisation de matériel courant, sauf dérogations justifiées par des circonstances exceptionnelles $; 3^{\circ}$ codifier, en les unifiant, les cahiers des charges aujourd'hui en usage sur les divers réseaux pour la fourniture des métaux et matières entrant dans la constitution du matériel [...]. »

Créée pour répondre à des fins de politique industrielle conjoncturelle, la commission, qui allait survivre au ministre Baudin, pose en fait les premiers jalons d'une politique structurelle d'unification ferroviaire nationale. Mais à tous petits pas : car les ingénieurs fonctionnaires du contrôle et les ingénieurs en chef responsables des services MT (Matériel et Traction) des sept grands réseaux devaient forcément arriver à s'entendre entre eux avant de retenir toute norme...

Dès le 31 mai 1901, les termes d'une première spécification pour la fourniture des tôles de chaudières étaient fixés et, en 1906, à l'issue de cette première étape d'unification, 22 spécifications étaient adoptées et mises en service après 29 mois de gestation en moyenne, visant les qualités métallurgiques de divers composants du matériel ${ }^{14}$.

Comme on le verra plus loin, la Grande Guerre allait constituer un contexte favorable à une vaste politique d'uniformisation, à une deuxième étape en particulier dans l'unification des cahiers des charges imposés aux fournisseurs. En juillet 1917, les ingénieurs des réseaux

14- Par exemple, essieux et bandages en acier, plaques de foyers, tubes à fumée et leurs viroles.

15- Voir Le Blant, « Note sur les unifications des spécifications techniques pour la fourniture des matériaux entrant dans la construction des voitures, machines et tenders des grands réseaux de chemins de fer français », Revue générale des chemins de fer, novembre 1922.

16- Séries normales de boulons, goujons, écrous, rondelles, goupilles, vis et rivets. Unification des chemins de fer (7 mars 1918), Béranger, 1919; 1920, 74 p.

17- Spécifications techniques et Cahiers des charges des Services du Matériel et de la Traction des grands réseaux de chemins de fer français, Béranger, 2 éd., 1919, 259 p. (40 spécifications). Sous la forme d'un petit Agenda Dunod (Recueil des cahiers des charges et spécifications techniques unifiés adoptés par les chemins de fer français), L. Violet du PLM publie en 1921 le recueil des 42 spécifications en vigueur, chronologiquement classées, depuis les boulons, goujons, vis en acier et écrous (mars 1918) jusqu'aux rondelles Grover (26 août 1920). 
s'attellent ainsi à réviser et à compléter les premières spécifications de 1901-1906 ${ }^{15}$. Une collection de 42 spécifications, achevée en mai 1920, donne lieu à diverses publications, partielles ${ }^{16}$ ou intégrales ${ }^{17}$.

Se plaçant à nouveau du point de vue des constructeurs ferroviaires français toujours aux abois au sortir de la guerre, ébauchant le programme de la reconstruction industrielle de la France, le fameux Rapport Clémentel ne manque pas de dicter aux compagnies des impératifs, au-delà de la prévision de leurs besoins quantitatifs en locomotives, voitures et wagons ${ }^{18}$ :

«Il faut, avant tout, que ces Compagnies favorisent nos industriels, qu'elles leur passent des commandes régulières, permettant un travail en série et qu'elles apportent dans les différents modèles une unification aussi grande que le permettent les emplois. »

En fait, les constructeurs français de matériel ferroviaire sont plus attachés à la régularité des commandes qu'à une certaine normalisation des fabrications. Par ailleurs, ils sont prêts à se servir de la normalisation technique pour verrouiller leur marché indigène, dès lors que celui-ci apparait menacé par des industries concurrentes. Ainsi, en 1933, la Chambre syndicale des constructeurs de matériel de chemins de fers'alerte de l'arrivée sur le marché des autorails des constructeurs et fournisseurs automobiles (Renault, Bugatti, Michelin, etc.), en quête de nouveaux débouchés. Un groupe de réflexion ad hoc propose une première riposte générale : «attirer l'attention des réseaux sur le tort considérable que leur fait la propagande de l'automobile et sur l'intérêt qu'ils ont à ne pas favoriser par leurs commandes d'aussi dangereux concurrents. »; mais il entend aussi, plus subtilement, ériger si nécessaire une barrière technologique : «Il y aurait le plus grand intérêt, pour lutter contre cette concurrence, à profiter de l'établissement par les réseaux de spécifications type, pour établir avec eux un contact étroit et permanent, susceptible de leur montrer la facilité avec laquelle les constructeurs attitrés de matériel ferroviaire s'adaptent à ces règles nouvelles, alors que les constructeurs automobiles, non habitués à des cahiers des charges, éprouvent des difficultés à en observer les prescriptions ${ }^{19}$. »

18- Rapport général sur l'industrie française, sa situation, son avenir, t. 1, Paris, Impr. nationale, 1919, p. 417.

19- Archives de la Fédération des industries ferroviaires, Groupe des autorails, $P V$ de la séance du 26 octobre 1933, intervention de M. de Geoffroy. C'est peu après, en janvier 1934, qu'est publiée, par la Commission d'études des automotrices des réseaux, la première Spécification technique pour la fourniture d'automotrices à moteurs thermiques, en 40 articles. 


\section{Des circonstances favorables à la normalisation}

S'il y a donc un plancher minimum de règles et contraintes uniformes qui a pu s'imposer à tout exploitant, des contextes particuliers s'avèrent favorables à de fortes pressions normalisatrices dans le domaine de la construction et de l'exploitation ferroviaires.

\section{Les leçons de l'exploitation en temps de guerre}

La Grande Guerre a servi de révélateur aux handicaps d'ordre militaire ou civil que crée le foisonnement de règlements et matériels ferroviaires propres à chaque réseau et, plus largement, l'absence de normes industrielles de base, telles que des pas de vis! Alors que les exigences logistiques des autorités militaires ignoraient en théorie les frontières techniques et administratives des réseaux, celles-ci s'avérèrent de puissants obstacles au transit d'un trafic militaire redoublé et devenu particulièrement essentiel. Bien entendu, alors qu'il était impensable de faire circuler sur des réseaux voisins des locomotives conçues «sur mesure » par les ingénieurs de chaque compagnie pour assurer tel trafic commercial sur telle ligne, les échanges de wagons, bien que plus faciles, restèrent limités : encore fallait-il assurer en effet une maintenance de premier niveau ubiquiste lorsque des avaries et incidents mineurs les immobilisaient hors de leur réseau d'attache. Ce qui supposait des pièces et outillages normalisés... Que ce soit dans le domaine industriel en général, ou dans le domaine ferroviaire, cette forte pression normalisatrice motiva des initiatives plutôt tardives ${ }^{20}$.

Signée du ministre des Travaux publics Claveille ${ }^{21}$, la lettre en date du 24 février 1918 qu'il adresse au directeur des Chemins de fer reflète la nouvelle doctrine de la standardisation. Après avoir évoqué les défis de l'après-guerre ("Il est indispensable de se préoccuper dès à présent des mesures à prendre après la guerre pour mettre à la hauteur des nécessités économiques l'organisme vital que constituent les réseaux de chemins de fer »), dans la perspective d'un programme décennal de

20- C'est par un décret du 11 juin 1918 que fut créée au ministère du Commerce une Commission de standardisation permanente, véritablement productrice de normes à partir de 1919. L'Association française de normalisation (AFNOR), créée en 1926 à l'initiative des industries électriques, prend ensuite le relais.

21- Sur le rôle déterminant de Claveille dans cette impulsion normalisatrice, voir G. Ribeill, « Du projet Claveille (1917) à la Convention Le Trocquer (1921). Le retournement libéral d'un projet de nationalisation du rail », in «Le statut des chemins de fer français et leurs rapports avec l'État, 1908-1982 », Revue d'histoire des chemins de fer hors série $\mathrm{n}^{\circ}$ 4, février 1996, p. 41 et sq. 
commandes de matériel roulant, l'enjeu de cette standardisation était ainsi souligné dans des termes vigoureux qui méritent d'être largement reproduits :

«Le matériel des chemins de fer français présentait avant la guerre une diversité regrettable, et aucune idée d'ensemble n'avait présidé au choix des types. La diversité dont il s'agit pouvait se constater, non seulement d'un réseau à l'autre, mais sur un même réseau, entre des véhicules destinés à un service identique.

Le moment est venu de réagir contre cette tendance. Lors des dernières commandes, un progrès a été déjà réalisé par l'adoption d'un type unique de wagons de 20 tonnes, auquel tous les réseaux se sont ralliés. Il est nécessaire d'entrer résolument dans cette voie, de façon à faciliter les commandes, la fabrication en série, et l'emploi sur chaque réseau du matériel appartenant aux réseaux voisins.

Il ne peut être question, bien entendu, d'arriver à l'uniformité générale des types de matériel sur tous les réseaux, chacun d'eux devant conserver son initiative et sa responsabilité et aucune entrave ne devant être apportée à une émulation qu'il serait dangereux de paralyser ; mais on doit tout au moins s'appliquer à ce que les types soient peu nombreux, à ce que chaque réseau profite des perfectionnements obtenus par ailleurs, et surtout à ce que la réparation des voitures et wagons de n'importe quel réseau devienne toujours possible sur n'importe quel autre. Faute de pièces et d'organes de rechange, on a constaté trop souvent des immobilisations anormales de véhicules sur des réseaux éloignés et il est essentiel que cet état de choses disparaisse à l'avenir. Les événements actuels ont démontré surabondamment l'intérêt qu'il y aurait à cet égard à arriver à ce que l'on appelé la "standardisation" du matériel, c'est-à-dire à l'adoption, dans la construction, de pièces de dimensions et de dispositions uniformes, et par suite interchangeables, comme l'ont admis certaines fabriques d'automobiles.

Les circonstances sont éminemment favorables à une mesure de ce genre, qui sera bien accueillie des constructeurs et du personnel, qui réduira les dépenses d'entretien, et qui assurera la bonne utilisation du matériel, en accélérant sa rotation. En effet, beaucoup de véhicules anciens seront à réformer ou à transformer, et les commandes à envisager porteront certainement sur un nombre d'unités très élevé. Rien ne sera plus facile en conséquence que de prévoir un nombre limité de types constitués à 
l'aide de pièces et d'organes semblables, dont des dépôts pourront être répartis sur tout le territoire, de manière à rendre les réparations très rapides.

Enfin, les types à adopter devront être étudiés d'après les données les plus récentes de l'expérience, en tenant compte de l'intérêt qu'offrent les wagons à tonnage élevé, des conditions particulières de la circulation sur les lignes où la traction électrique sera installée, et de la nécessité, au point de vue du confortable des voyageurs, de conserver au matériel français la réputation dont il jouit à l'étranger. »

De mêmes impératifs visaient les réseaux d'intérêt local où, compte tenu du choix du matériel abandonné aux autorités locales, « il arrivait trop fréquemment que le matériel d'une ligne déterminée ou d'un petit réseau ne pouvait circuler sur les réseaux voisins ». Dans les régions libérées notamment, la reconstitution de leurs lignes devait donc être orientée d'une part vers "la réduction du nombre de types, en généralisant ceux que l'expérience aura montrés les plus avantageux [...] afin de faciliter le circulation d'un réseau à l'autre », d'autre part vers la «fabrication en série ».

Claveille avait jeté aussi les bases d'un autre programme décennal concernant le matériel fixe, aux fins de réduire des différences entre rails "peu appréciables d'un réseau à l'autre pour un poids unitaire comparable » et d'entreprendre la standardisation du petit matériel de la voie (tire-fond, boulons, selles, etc.) ${ }^{22}$.

Plus largement encore, l'exploitation devait être aussi conçue sur de nouvelles bases, avec des règlements de réseau uniformisés dans la limite du possible, ainsi que leur signalisation. Et si « les efforts tentés à de fréquentes reprises pour arriver à un régime plus rationnel sont restés infructueux ", la réforme de la signalisation méritait au premier chef «la persévérance voulue pour la faire aboutir», compte tenu de son intérêt évident, jugeait Claveille. De même, il préconisait une « refonte générale et rationnelle de la tarification des grands réseaux » tendant à faire « comme si les frontières entre les Réseaux étaient supprimées et si

22- Voir Frœbé, ingénieur du Matériel fixe aux Chemins de fer de l'État, « Note sur la standardisation du matériel des voies des chemins de fer français », Revue générale des chemins de fer, novembre 1920.

23- Malgré le freinage des compagnies, cette impulsion allait aboutir en 1920 à l'institution d'un statut des agents et d'une grille des rémunérations communs à tous les grands réseaux, à l'origine du statut actuel des cheminots. 
l'ensemble des chemins de fer étaient placés sous la direction d'une Administration unique ayant exclusivement en vue les intérêts généraux du Pays ».

Enfin, du rapprochement des diverses réglementations en cours et propres à chaque réseau concernant le recrutement, l'avancement et la rémunération de leurs agents, on devait aboutir aux améliorations à leur apporter dans le sens de leur harmonisation et unification ${ }^{23}$. De manière plus explicite, les leaders syndicalistes réformistes partageaient les critiques faites aux compagnies ${ }^{24}$, jugées éminemment responsables de la crise des transports qui se prolonge encore après la fin de la guerre ${ }^{25}$.

Cette vigoureuse impulsion gouvernementale allait voir ses effets durer quelques années. Timidement engagées avant-guerre, les perspectives de développement de la traction électrique motivèrent notamment la création en 1918 d'une commission chargée de sélectionner la future tension standard qui devrait être adoptée par tous les grands réseaux : elle allait conclure à l'option du $1500 \mathrm{~V}$ continu, érigé donc en norme officielle nationale ${ }^{26}$. En 1919, puis en 1925, confrontés au renouvellement de leurs anciens rails - plus de 60 modèles en tout -, les réseaux étudient une gamme de 4 types de rails $(26 \mathrm{~kg}, 36 \mathrm{~kg}$, $46 \mathrm{~kg}$ et $55 \mathrm{~kg}$ au mètre) aux profils unifiés. Un cahier des charges unifié pour la fourniture des rails est mis en application fin 1923.

24- En ce qui concerne les machines, « il y avait impossibilité d'organiser le progrès, car les Compagnies, jalouses les unes des autres, étaient incapables de coordonner leurs efforts pour la recherche d'améliorations et, en fait, subissaient le progrès bien plus qu'elles ne le provoquaient ", énonce sévèrement Marcel Bidegaray, responsable de la Fédération CGT des cheminots (Les Chemins de fer en France. L'Exploitation d'aujourd'hui par les compagnies. L'Exploitation de demain par la nationalisation des chemins de fer, 1919, p. 45-46).

25- « La crise des transports n'est due qu'à ce défaut d'organisation : étanchéité complète entre les réseaux, manque absolu de coordination entre eux pour l'œuvre commune, qui était la défense nationale », ibid., p. 45.

26- Voir Christophe Bouneau, «Un débat technique national : l'électrification des chemins de fer en France au début de l'entre-deux-guerres ", in « Les chemins de fer, l'espace et la société en France », actes du $1^{\text {er }}$ colloque de l'AHICF, Paris, mai 1989, Revue d'histoire des chemins de fer hors série $\mathrm{n}^{\circ}$ 1, p. 151 et sq. Voir aussi, avec une vision plus panoramique et comparative, Jean-François Picard, «Technique universelle ou filières nationales ? Le cas de l'électrification des chemins de fer en Europe », Sciences et technologie en perspective, Université de Nantes, $1^{\text {er }}$ trimestre 1996.

27- Le Nord et l'Est ne s'associeront qu'en 1929. 
Un Office central d'études du matériel (OCEM) est créé en octobre 1919 qui associe trois compagnies (PLM, PO, Midi) ${ }^{27}$ et les deux réseaux d'Etat (ex-Ouest et Alsace-Lorraine) : de ce bureau d'études doivent sortir les premiers types communs de matériel roulant à plusieurs réseaux, formant donc de grandes séries d'un coût de fabrication plus économique. Ce sera le cas d'une série de wagons de 20 tonnes à deux essieux et entraxe de 4,5 m, modulés en tombereaux, plats et couverts, avec ou sans guérites fermées à plate-forme basse, pouvant être équipés du frein continu; soit une conception intégrant pour la première fois des normes et spécifications promues par l'UT (Unité technique des chemins de fer) et par l'UIC. Un tel matériel moderne, pouvant « assurer pendant de longues années le trafic intérieur comme le transit avec l'étranger », consacrait ainsi le premier « début dans l'unification du matériel européen $»^{28}$. De fait, les ingénieurs du Matériel des compagnies répugnèrent largement à transférer la conception de leur parc à l'OCEM dont le bilan demeure médiocre, jusqu'à sa disparition ${ }^{29}$.

A l'instigation du directeur du contrôle technique des chemins de fer, l'ingénieur des Mines Maison, une commission fut chargée d’une réforme du Code des signaux, que présida Verlant, directeur de l'exploitation du PLM. Agréée en deux temps, en 1932 et 1934, la réforme se traduisit essentiellement par l'adoption du feu vert pour indiquer la voie libre et celle du feu jaune en remplacement du feu vert d'avertissement ou de ralentissement. Sa mise en application progressive en six étapes s'échelonna d'avril 1935 à décembre 1936. Mais lorsque la SNCF fut créée, il restait encore à unifier la signification des signaux et leurs conditions techniques d'emploi ${ }^{30}$. Certaines particularités réglementaires et technologiques héritées des anciens réseaux furent conservées, du fait de leur trop grande étendue, par exemple en matière de signaux de

28- Voir l'article de Vallancien, ingénieur principal à l'OCEM, «Unification du matériel roulant de petite vitesse des grands réseaux français. Wagons de 20 t. à deux essieux avec entr'axe de 4,500 m (1929) ", Revue générale des chemins de fer, septembre 1929, p. 242-252.

29- A la veille de la guerre, le parc roulant (voitures et wagons) de la SNCF était constitué pour $54 \%$ de matériel régional hérité des 7 anciens réseaux, pour $32 \%$ de matériel commun et seulement pour $14 \%$ de matériel unifiécommandé auprès de l'OCEM (voir Arnaud, « Normalisation des organes de roulement de voitures et wagons », Revue générale des chemins de fer, janvier-février 1945).

30- Voir la brochure de M. Walter, éditée par la SNCF, Unification et simplification des procédés de signalisation et de transmission, Conférence faite le 21 mai $1943 \mathrm{au}$ Centre d'études supérieures des transports.

31- Sur l'histoire de cette évolution de la signalisation, voir Alain Gernigon, Histoire de la signalisation ferroviaire française, Paris, La Vie du Rail, 1998, p. 94-121. 
blocks. Et ce n'est qu'après-guerre, en 1947, que la SNCF peut éditer un Règlement général de sécurité dont le premier fascicule (son Titre I) sera consacré aux signaux ${ }^{31}$.

\section{Le nouveau cours d'unification de la SNCF}

De la création (d'origine politique) de la SNCF en 1937 résultent à la fois la fin des frontières inter-réseaux et la juxtaposition de systèmes et matériels d'exploitation fort différents. Par exemple, le parc des engins thermiques, développé à partir de 1931, avait mobilisé de nombreux constructeurs, auxquels la plus grande liberté d'initiative avait été laissée par les réseaux, afin de tirer parti au mieux de cette nouvelle émulation technologique et de profiter au maximum des potentialités de ce nouveau mode de traction. D'où un parc hérité par la SNCF extrêmement hétérogène et d'exploitation fort coûteuse ${ }^{32}$.

L'entreprise publique engage donc un nouveau cours de réduction des types hérités, d'unification organique de l'exploitation et de normalisation des matériels, associés aux impératifs d'économies que lui assigne son nouvel État-patron. Dès janvier 1938, il est créé à la SNCF un Bureau de normalisation et d'unification (BNU), chargé de développer en son sein une politique de normalisation technique des pièces et matières en usage courant et de représenter l'entreprise dans les commissions de travail des organismes spécialisés nationaux ou internationaux (AFNOR, CSNOR, ISA, etc.). Le bilan établi à la veille de la Deuxième Guerre mondiale apparait toutefois modeste, limité à de simples et petites pièces de détail du matériel roulant ou à l'outillage des ateliers, plutôt qu'à des composants plus lourds tels que boites d'essieux, corps de roues, essieux, etc. ${ }^{33}$. Et c'est seulement durant les années de guerre que les bureaux du Matériel entreprennent la normalisation des organes de roulement (137 types d'essieux, 61 types de bandages, 165 types de coussinets, etc.) ${ }^{34}$. Créé en 1934 à Saint-Ouen dans le sillage de l'adoption du code unifié Verlant, le laboratoire central de signalisation, dont hérite la SNCF, s'attelle à la normalisation des signaux lumineux ${ }^{35}$. La

32- Voir C. Tourneur, « La normalisation dans les matériels de traction à moteurs thermiques », Courrier de la normalisation, $\mathrm{n}^{\circ} 149$ (septembre-octobre 1959).

33- Chatel, "La normalisation et l'unification à la SNCF », Revue générale des chemins de fer, juillet 1939.

34- Arnaud, « Normalisation des organes de roulement de voitures et wagons », Revue générale des chemins de fer, janvier-février 1945.

35- M. Walter, conférence citée.

36- R. Tarbouriech, «Le Bureau de Normalisation des Chemins de Fer (BNC) », Courrier de la normalisation, $\mathrm{n}^{\circ} 149$ (septembre-octobre 1959). 
combinaison de 14 éléments unifiés allait permettre de traiter autant de cas que les 200 modèles antérieurs de panneaux lumineux et les modèles de lampes étaient réduits de plus de 100 types à 46, puis à $17 \ldots$

Durant l'Occupation, en vertu d'un décret du 24 mai 1941 impliquant les deux comités d'organisation du matériel ferroviaire (MATFER) et des voies ferrées d'intérêt local (COVFIL), le BNU étend ses compétences en dehors de la SNCF, transformé en 1943 en Bureau de normalisation des chemins de fer (BNCF) dès lors qu'était apparue souhaitable une certaine normalisation de tous les chemins de fer français, qu'ils soient à voie normale ou étroite, d'intérêt général ou local, en métropole ou outre-mer ${ }^{36}$.

Sous l'impulsion du directeur général Le Besnerais, les bureaux d'études de la traction de la SNCF sont invités à définir quelques « types unifiés d'avenir» qui seront donc commandés en grande série. Le temps de la locomotive conçue «sur mesure » est clos, même s'il faut compter encore avec quelques « coups déjà partis » mais encore dans les cartons, antérieurs à cette nouvelle politique drastique que plusieurs types unifiés de locomotives symbolisent bien, $141 \mathrm{P}$ et $150 \mathrm{P}$ tout particulièrement $^{37}$. Après-guerre, cette politique est poursuivie : 4 types d'autorails sont retenus ( $90 \mathrm{ch}, 150 \mathrm{ch}, 300 \mathrm{ch}$ et $600 \mathrm{ch}$ ), ainsi qu'un type unique de remorque avec simplement deux aménagements possibles $\left(1^{\text {re }} /\right.$ $2^{\mathrm{e}}$ classe et $2^{\mathrm{e}}$ classe/fourgon). De même, s'agissant des locotracteurs, deux types doivent remplacer les 40 types hérités des anciens réseaux.

Les commandes aux États-Unis et au Canada d'un nouveau modèle de locomotive construite en grande série, la fameuse Mikado 141-R à simple expansion, marquent un tournant décisif, puisque dans le sillage de ces très grands séries d'engins moteurs (1 340 locomotives commandées en deux tranches, réparties en « charbonnières » et locomotives chauffées au fuel), à la conception simplifiée à l'américaine et à la conduite banalisée, est associé un entretien en grande série "à la chaîne ", véritable cheval de Troie du taylorisme dans les ateliers de la SNCF ${ }^{38}$.

37- Voir les récents ouvrages consacrés à ces types unifiés de locomotives de la SNCF et signés de Bernard Collardey et d'André Rasserie : Les 141 P, ces valeureuses Mikado, Paris, La Vie du Rail, 1999 ; Les Locomotives à vapeur unifiées 241 P, 240 P, 150 P, Paris, La Vie du Rail, 2001 ; Les Locomotives à vapeur unifiées (2), 232 R, 232 S, 151 TQ, 050 TQ et les prototypes, Paris, La Vie du Rail, 2002.

38- Bernard Collardey et André Rasserie, Les 141-R, ces belles américaines, Paris, La Vie du Rail, 1981 (rééd. 1995) ; sur les effet sociotechniques induits, G. Ribeill, Les Cheminots, Paris, La Découverte, 1984, p. 54 et sq. 
En matière de traction électrique, on sait que c'est le $1500 \mathrm{~V}$ continu qui est retenu encore pour l'électrification de la ligne impériale, entre Paris et Lyon, avec deux premiers types de locomotives originaux, BB 8100 et 2D2. Mais de nouveaux et rapides progrès justifient une nouvelle série de CC 7100 plus légères ; puis une série de BB 9200, encore plus légères... C'est l'impératif d'économie imposé à nouveau à la SNCF qui motive, d'une part, le développement sur la ligne du NordEst à électrifier du nouveau courant monophasé $25 \mathrm{kV} / 50 \mathrm{~Hz}$, d'autre part le souci de pouvoir tester quatre conceptions différentes d'engins moteurs (BB 12000, BB 13000, CC 14000 et CC 14100) : le surcoût sera compensé par le recours à des organes mécaniques identiques (bogies, caisse, cabine de conduite unique centrale, pupitre de commande) et à des circuits conçus selon des schémas types de montage identiques. Soit une conception modulaire, souple mais économique, de matériel nouveau, unifiée au maximum, sollicitant de la part des conducteursélectriciens, quel que soit le type d'engin conduit, de mêmes gestes et actes réflexes pour les opérations de conduite et de dépannage, ou requérant de mêmes procédures d'entretien chez les ouvriers des dépôts et ateliers ${ }^{39}$.

\section{Les facteurs de résistance à la normalisation}

Malgré ces facteurs permanents ou conjoncturels favorables à la normalisation, les facteurs de résistance, endogènes à la culture techniciste des ingénieurs ferroviaires français, propres aussi à une certaine émulation naturelle entre réseaux, leur ont toujours fait contrepoids. Dans un article ancien ${ }^{40}$, François Caron brossait ce portrait de l'ingénieur français hostile à la norme anti-progrès :

«Les ingénieurs français ont toujours considéré la locomotive comme un objet d'art indéfiniment perfectible, peu susceptible par conséquent d'être soumis à des standardisations contraignantes. Pour eux, il y avait contradiction entre la standardisation et la production de masse, d'une part, et le progrès technique, d'autre part, qui risquait d'être bloqué par elles. Ils ne voulaient pas être condamnés à employer toujours indéfiniment le même modèle. »

39- F. Nouvion, «Locomotives électriques et normalisation », Courrier de la normalisation, $\mathrm{n}^{\circ} 149$ (septembre-octobre 1959).

40- F. Caron, « Le rôle des compagnies de chemins de fer en France dans l'introduction et la diffusion des procédés Bessemer », in L'Acquisition des techniquespar les pays non-initiateurs, Paris, Éd. du CNRS, 1973, p. 570.

41- F. Jacqmin, De l'exploitation des chemins de fer, Paris, Garnier, 1868, t. 1, p. 131-132. 
Attitude confirmée par les propos mêmes de certains d'entre eux. Ecoutons le directeur de l'exploitation de l'Est, François Jacqmin, réfuter en 1868 le principe d'uniformisation des règlements techniques ${ }^{41}$ :

"L'uniformité absolue des règlements entraînerait un inconvénient très grave ; elle interdirait à l'avance toute étude, tout progrès. L'exploitation des chemins de fer est trop récente pour que l'on puisse la considérer comme parfaite, et on doit encore compter sur de nouveaux perfectionnements [...]. La diversité dans les détails est donc favorable au progrès [...]. »

Les conditions spécifiques d'exploitation justifient même des particularismes techniques et des accoutumances professionnelles, que leur transformation doit préserver de tout bouleversement ultérieur :

«Chaque réseau se trouve, au point de vue de la sécurité, placé dans des conditions spéciales auxquelles il serait dangereux de porter atteinte ; dans des services compliqués comme ceux de la banlieue de Paris, il existe un ensemble de signaux consacrés par une expérience de près de 30 années, et personne ne voudrait prendre la responsabilité qu'entrainerait le moindre trouble porté à cette organisation. »

Parmi ces dispositions qu'il faut donc conserver bien qu'elles n'aient d'autre valeur que celle de «l'ancienneté », Jacqmin évoque notamment la conduite à droite sur la grande artère alsacienne :

«Sur toutes les lignes qui partent de Paris, on a pris pour voie de départ la voie située à gauche en entrant dans la gare, et ce système a été naturellement suivi sur toutes lignes françaises ; mais il existe une exception : sur la ligne de Strasbourg à Bâle, ouverte à l'exploitation en 1839, on avait pris pour voie de départ la voie droite. Lorsque la jonction de cette ligne ancienne avec les lignes partant de Paris a été faite, on s'est trouvé dans une grande perplexité et on pensé que ce qu'il y avait de plus simple était de changer sur la ligne de Strasbourg-Bâle le sens de la marche des trains. Théoriquement, rien ne semblait plus facile ; pratiquement, il a fallu reculer devant tous les obstacles qui se sont présentés, et l'administration supérieure a dû sanctionner par une décision spéciale une exception à la règle, en apparence si simple, du sens de la circulation des trains. »

42- Cas, au Nord, d'Asselin, abandonnant les prometteuses locomotives Baltic mises au point par son prédécesseur, Du Bousquet (voir B. Escudié et J. Payen, Notes et traduction de H. C.B. Rogers, André Chapelon (1892-1978), Paris, CNRS Éditions, 1992, p. 55). 
Lucide, le ministre Baudin, dans sa circulaire précitée du 18 août 1900, entendait bien mettre un terme aux excès bien analysés et dénoncés d'une émulation qui abritait parfois l'orgueil têtu ou le souci de distinction personnelle d'un ingénieur par rapport à ses pairs des autres réseaux ou, pire, à son prédécesseur dans la même compagnie ${ }^{42}$ :

«Sans doute, la marche du progrès est incessante, et il ne peut être question de confiner les Compagnies de chemins de fer dans l'emploi d'un certain nombre de types de locomotives, quelque judicieusement choisis qu'ils puissent être. Ce serait fermer la porte à toutes les recherches et à toutes les améliorations. Mais il n'est pas non plus indispensable que chaque chef de traction mette, pour ainsi dire, sa griffe personnelle sur les projets qui émanent de ses services et s'ingénie à faire autrement que ses collègues des autres réseaux quand il n'a pas, à l'appui des modifications qu'il présente, des raisons péremptoires à faire valoir. Il importe peu, par exemple, du point de vue des services qu'elles peuvent rendre, que deux machines diffèrent par les congés ou les évidements des bielles, la forme des ailettes des tubes, etc. Mais ces détails ont, au contraire, une grande importance quand il s'agit de couler des pièces ou d'établir des mandrins. »

Mais inversement, après-guerre, avocat des compagnies et idéologue tout dévoué, l'ingénieur fonctionnaire Colson entendait réfuter le reproche, fait au régime d'exploitation français, d'un " manque d'unité » et de "divergences parfois inexplicables entre le régime des tarifs et entre les types de matériel dans les différents réseaux $»^{43}$ :

"Aujourd'hui tout le monde vante la standardisation, employant un mot français déplorable pour exprimer une idée qui ne l'est pas moins, car il représente la mort du progrès, tout simplement. Pour adopter un type uniforme en chaque matière, un type qu'on pourra changer qu'en mettant d'accord 6 ou 7 réseaux, il faut supposer qu'on connait la perfection. Je crois que ce moment n'est pas encore venu. »

En fait, entre les boulons et les locomotives, le jugement de Colson est plus contrasté :

«On a eu le tort, dans les chemins de fer, de ne pas arriver plus tôt à la standardisation de certains détails, du type des pas de vis, des boulons, des pièces de rechange ; mais quant à la standardi-

43- Colson, "Nos chemins de fer", in L'Outillage économique de la France, Paris, Alcan, 1921, p. 72-73.

44- Voir L. Rimbaud, «La normalisation dans les voitures et les wagons », Courrier de la normalisation, $\mathrm{n}^{\circ} 149$ (septembre-octobre 1959), p. 485. 
sation des types de machines, $j$ 'estime que la diversité est une chance de progrès. Quand il y a six ingénieurs en chef du matériel, six chefs d'exploitation commerciale, il y a plus de chances pour qu'une amélioration se produise que quand il n'y en a qu'un, et quand l'un a réalisé un progrès sérieux, il est suivi par les autres, parce que le progrès s'impose par ses résultats. »

Et en 1959 encore, du côté des ingénieurs de la SNCF, résonnait toujours le même son de cloche ${ }^{44}$ :

"L'ingénieur est sollicité sans cesse par deux tendances opposées : l'une l'incite à tirer profit sans délai des progrès techniques généraux ou du fruit de ses propres recherches pour créer du nouveau susceptible d'agir favorablement sur la clientèle et d'accroitre la vente, l'autre le conduit au contraire à freiner prudemment cette inclination pour éviter la prolifération des modèles qui risque d'obérer les prix de revient, aussi bien ceux de la fabrication que ceux de l'entretien. » En 1945, l'ingénieur en chef du Matériel Maurice Bailleul avait d'ailleurs proposé une curieuse solution pour résoudre la tension croissante entre unification conquérante et innovation bridée, à savoir la mise en œuvre conjointe de programmes décennaux de recherche et de construction, menés en parallèle mais s'ignorant durant une période de dix ans, solution ainsi justifiée $e^{45}$ :

« Si "unifier" signifie "se limiter à des types bien déterminés", cela ne doit pas avoir pour conséquence de stopper la marche du progrès et de se priver délibérément des avantages qu'il permet d'obtenir. Comment, dès lors, concilier ces deux points de vue ? La solution et simple.

Les programmes de construction, établis en fonction des besoins des années à venir, sont arrêtés ne varietur pour une certaine période, dix ans par exemple. Autrement dit, on prend l'engagement, pendant cette période, de construire, et de construire seulement, les véhicules étudiés conformément aux indications ci-dessus. Ceci permettra la mise en chantier de grandes séries, c'est-à-dire de construire dans des conditions particulièrement avantageuses. La diversité des pièces de rechange sera, d'autre part, réduite ipso facto le plus possible.

45- Maurice Bailleul, « Unification et normalisation du matériel des chemins de fer », in Le Matériel roulant des chemins de fer français, Paris, Éd. Eyrolles, 1945, p. 263-266. 
Mais pendant ce temps, les bureaux d'études ne chômeront pas, bien au contraire. Et leur activité se portera sur l'étude et la mise au point de prototypes, pourvus des derniers perfectionnements de la technique, en vue de la préparation des nouveaux types de matériels à commander pendant la période décennale suivante. Ainsi, le progrès continuera sa marche inéluctable, mais il se manifestera non d'une façon progressive (ce qui serait désastreux par les bouleversements incessants apportés dans les programmes constructifs), mais par bonds successifs, à la fin du palier que constituera chaque programme décennal. »

Une solution originale, mais fort peu réaliste !

\section{Sur la longue durée, un bilan défavorable à l'intégration ferroviaire}

A l'heure d'une intégration croissante, impérative, des réseaux dans une Europe pacifiée depuis 1945 puis libéralisée par le Traité de Rome, l'ancien président de la SNCF puis secrétaire général de l'UIC, Louis Armand, avait milité au premier rang pour cette "Européisation des chemins de fer». Mais, à la suite d'une fort lucide analyse critique du passé, il tenait pourtant à l'adresse de sa " patrie professionnelle » du rail des propos franchement amers et désabusés, dans des écrits posthumes il est vrai, parus quatre ans après sa mort.

Dans la première étape du développement de la vocation européenne des réseaux, durant la première moitié du $\mathrm{xx}^{\mathrm{e}}$ siècle, « les rapports des réseaux et de l'Europe furent circonspects et même plutôt mous ", pour deux raisons ${ }^{46}$ :

«D'abord les organisations internationales du chemin de fer furent trop souvent tiraillées entre les prétentions à l'hégémonie des réseaux français d'un côté, des chemins de fer allemands de l'autre. Elles ont dû louvoyer pour éviter des affrontements déclarés dont les causes dépassaient de loin les problèmes du rail [...]. »

Seconde raison, " une place un peu trop grande a été faite aux hommes de droit dans les premiers conseils internationaux » : des compétences qui ont plus de peine que celles d'ingénieurs «à s'évader du complexe de Babel », à se familiariser avec l'universel, introductrices par exemple dans ces «pandectes du rail » qu'est la Convention de Berne de «trop de verrous, de ferrures », d'interdictions diverses d'ordre commercial, critiquait Louis Armand.

46- L. Armand, Message pour ma patrie professionnelle, s.l., Les Amis de Louis Armand, 1974, p. 85.

47- Ibid., p. 90.

48- Ibid., p. 90 ; p. 92. 
Durant la seconde étape de l'européisation ouverte après-guerre, les clivages ferroviaires nationaux devaient être surmontés : «les travailleurs de l'Europe du rail ont à obtenir d'eux-mêmes un détachement de leurs liens affectifs avec leur réseau d'origine, susceptible d'altérer l'objectivité de leur jugement et de leur attitude vis-à-vis des autres. » Cela afin d'acquérir « une sorte d'extra-territorialité de comportement, tâche d'autant plus difficile en vérité que presque partout, maintenant, les locomotives circulent sous le pavillon national $»^{47}$. Et d'évoquer les vices même de fonctionnement d'une Union internationale des chemins de fer par trop bureaucratique, « dont le nom a été trop longtemps en avance sur la signification des travaux $»^{48}$ :

«Si l'on a pu se mettre d'accord, après d'épuisantes discussions, sur l'âge jusqu'auquel les enfants paieraient demi-place, on n'a pas encore réussi à établir de véritables tarifs internationaux. Tout se passe comme s'il était plus aisé de concilier les services "dépensiers" que les services "encaisseurs". »

Malgré tout, sans doute le wagon EUROP fut-il l'une des plus belles réussites de l'UIC. Après-guerre, les réseaux membres s'étaient engagés dans un programme de wagons unifiés qui, pour être aptes au trafic international, devaient satisfaire un ensemble de conditions, notamment l'équipement d'un certain nombre d'organes à la fois interchangeables et disponibles dans tous les pays. Confiée à l'Office de recherches et d'essais de l'UIC (ORE, aujourd'hui ERRI), la standardisation de ces wagons allait permettre d'aboutir à un seul type de tombereau, de plat et de couvert, le marquage EUROP leur servant de laissez-passer aux frontières. Après ces wagons unifiés, la création en 1956, à Bâle, d'Eurofima, la «banque des wagons », puis leur numérotation uniforme adoptée par tous les chemins de fer de l'Europe et de l'URSS ${ }^{49}$, marquent sûrement des étapes importantes vers des échanges facilités et intensifiés.

Si les réseaux nationaux ont été parfois coupables de préjudiciables concurrences, les politiques industrielles protectionnistes des gouvernements les ont souvent entretenues ou amplifiées : «Le plus souvent, les réseaux furent embrigadés dans des rivalités inter-étatiques venant entraver, explicitement ou sans l'avouer, les ententes que les ingénieurs du rail profilaient sur l'Europe ferroviaire ${ }^{50}$. »

49- La Convention stipulant la numérotation des wagons est signée en 1964 entre l'UIC et l'OSJD (organisation ferroviaire internationale des pays de l'Est).

50- Ibid., p. 93.

51- Ibid., p. 95. 
Parfois, ces rivalités opposèrent de vastes alliances « patriotiques » regroupant État, exploitant et industriels ferroviaires et voulant imposer cette hégémonie que dénonçait L. Armand : en témoigne la partie de bras-de-fer franco-allemande en matière de normes d'électrification ferroviaire qu'a jouée pourtant L. Armand lui-même durant les années 1950-1960, promoteur naturel et universel de son fameux courant de traction industriel.

C'est pourquoi, en 1974, le paysage ferroviaire européen est très loin encore de cette Société internationale des chemins de fer européens qu'avait appelée pourtant de tous ses vœux Louis Armand ${ }^{51}$ :

«Les dirigeants du rail ont été, à l'exemple de politiques, placés devant un choix : on s'associe ou l'on s'intègre. On s'arrête à l'Europe des réseaux comme à celle des patries, ou l'on pousse jusqu'à édifier progressivement une sorte de Société internationale des chemins de fer européens. J'ai la conviction que le rail doit opter en faveur de la seconde solution, même s'il prend ainsi une position d'avant-garde, même s'il assume les risques encourus par tous ceux qui vont patrouiller devant le gros des troupes. »

Malgré les bonnes volontés affirmées de quelques dirigeants ferroviaires et l'extrême pression amplifiée depuis les années 1990 de la Commission européenne, l'Europe ferroviaire intégrée demeure toujours largement une utopie, tant persistent en fin de compte les facteurs favorables à la différenciation et à la distinction ferroviaires face à une volonté normalisatrice émiettée, diluée, difficile aussi à mettre en œuvre ${ }^{52} \ldots$ Faut-il évoquer les récentes batailles franco-allemandes en matière de système de contrôle-commande sécuritaire des circulations ferroviaires (projets ASTREE/DIBMOF) ou de trains à grande vitesse (systèmes TGV/ICE), fussent-elles bien sûr parfois menées à fleuret moucheté sous la bannière des accords de coopération DEUFRAKO ${ }^{53}$ ?

52- Cf. Philippe Bruel, La Normalisation ferroviaire européenne. Rapport de synthèse, février 1995, p. 107-108: « La caractéristique des travaux de normalisation ferroviaire européenne est le foisonnement (rappelons qu'il n'existe pas de schéma directeur normatif rédigé). Il y a plus d'une centaine de projets, sans cohérence ou sans traçabilité d'ensemble. » L'expert souligne les difficultés intrinsèques de la normalisation des systèmes ferroviaires, par le mélange extrême de normes mécaniques, électriques, informatiques et de télécommunications.

53- Voir la plaquette DEUFR AKO, 1978-1998.

54- F. Caron, «La naissance d'un système technique à grande échelle. Le chemin de fer en France (1832-1870) », Annales HES, juillet 1998. 
En conclusion, si l'on fait un bilan de l'antagonisme entre les processus d'unification et de différenciation ferroviaires, il est patent que les seconds l'ont emporté structurellement sur les premiers, en dépit des avantages économiques et industriels couramment reconnus à la normalisation, ou des facilités d'échanges et de coopération entre réseaux ferroviaires qui en résultent. Alors que ce sont des facteurs ou forces exogènes aux exploitants qui les ont poussés à s'engager sur les voies de cette normalisation, pour de multiples raisons, les exploitants ont toujours eu le dernier mot pour justifier et imposer leurs « normes singulières ».

Dans un récent article stimulant ${ }^{54}$, F. Caron, formulant une modélisation des processus de différenciation des options techniques ferroviaires en France au Xix ${ }^{e}$ siècle, s'en tenait d'ailleurs à trois types d'explication, relevant tous du champ autonome des compagnies de chemins de fer et de leurs initiatives :

- selon le processus de différenciation proliférante, toute norme technique est antinomique du progrès, peut-on résumer. Nous avons longuement illustré plus haut la récurrence de cet argument d'ingénieur ;

- selon le processus de différenciation fonctionnelle, chaque système technique doit s'adapter à son propre environnement, tel contexte géographique par exemple (autre argument d'ingénieur), ou à son segment de marché, telle desserte ou trafic commercial par exemple (argument de marketing);

- selon le processus de différenciation identitaire, dans un souci de distinction/affirmation de son originalité, chaque système porte l'empreinte délibérée de sa culture d'entreprise, alors que son dirigeant amplifie sa marque de fabrique.

Dans ce même cas des compagnies françaises, nous avons nousmême récemment rappelé l'importance et la variété des processus de différenciation à l'œuvre qui relèvent de leur culture d'entreprise ferroviaire $^{55}$. Et lorsque ces compagnies se fondent dans des entreprises nationales unifiées - la SNCF dans le cas français -, les faits développés plus haut révèlent la persistance de ce facteur de différenciation technique déployé maintenant à l'échelle internationale et qu'amplifie même parfois la politique des tutelles gouvernementales, protectionnistes chez

55- Cf. G. Ribeill, « Variations sur les cultures d'entreprise : le cas des compagnies françaises de chemins de fer », in M. Merger et D. Barjot, avec la coll. de M.-N. Polino(sous la dir. de), Les Entreprises et leurs réseaux : hommes, capitaux, techniques et pouvoirs, XIX'-XX' siècles, Mélanges en l'honneur de François Caron, Paris, Presses de l'Université de Paris-Sorbnne, 1998. 
elles mais conquérantes hors des frontières. Les bonnes raisons de ne pas normaliser, de se distinguer ou de s'isoler par des innovations singulières, ont toujours de beaux jours devant elles...

\section{Annexe 1}

\section{Les principales organisations ferroviaires internationales}

\begin{tabular}{|l}
\hline \multicolumn{1}{c}{ Institutions } \\
\hline Conférence européenne des horaires (CEH) \\
\hline $\begin{array}{l}\text { Conférence internationale pour l'Unité technique des } \\
\text { chemins de fer (UT) }\end{array}$ \\
\hline $\begin{array}{l}\text { Association internationale du congrès des chemins } \\
\text { de fer (AICCF) }\end{array}$ \\
\hline $\begin{array}{l}\text { Convention internationale concernant le transport des } \\
\text { marchandises par chemins de fer (CIM) }\end{array}$ \\
\hline $\begin{array}{l}\text { Comité international des transports par chemins de } \\
\text { fer (CIT) }\end{array}$ \\
\hline Union internationale des wagons (RIV) \\
\hline Union internationale des voitures et fourgons (RIC) \\
\hline Union internationale des chemins de fer (UIC) \\
\hline $\begin{array}{l}\text { Convention internationale concernant le transport des } \\
\text { voyageurs et des bagages par chemins de fer (CIV) }\end{array}$ \\
\hline $\begin{array}{l}\text { Conférence internationale des horaires des trains de } \\
\text { marchandises (LIM) }\end{array}$ \\
\hline Bureau central de compensation (BCC) \\
\hline Bureau international des containers (BIC) \\
\hline Comité des transports intérieurs de l'ONU (CTI) \\
\hline - . \\
\hline
\end{tabular}




\begin{abstract}
Annexe 2
Questions relatives à la normalisation, à la coordination et à l'interopérabilité des réseaux, traitées au Congrès international des chemins de fer (1885-1966)
\end{abstract}

Domaine : M : Matériel ; EX : Exploitation ; VT : Voie et travaux ; OG : Ordre général ; $\mathrm{CFE}$ : Chemins de fer économiques (et coloniaux).

Q. : question, suivie de son $\mathrm{n}^{\circ}$ en chiffres romains.

\begin{tabular}{|c|c|c|}
\hline Congrès & $\begin{array}{l}\text { Domaine } \\
\text { Question } \\
\text { (numéro) }\end{array}$ & Sujet \\
\hline $\begin{array}{l}\text { 1885(I) } \\
\text { BRUXELLES }\end{array}$ & M, Q. III & $\begin{array}{l}\text { Construction du mé } \\
\text { vue d'en faciliter l'é }\end{array}$ \\
\hline Ibid. & EX, Q. IX & $\begin{array}{l}\text { Gares communes, } \\
\text { services d'échang€ }\end{array}$ \\
\hline 1887 (II) MILAN & OG, Q. XXIV & $\begin{array}{l}\text { Relations internatio } \\
\text { administrations de }\end{array}$ \\
\hline Ibid. & $\begin{array}{l}\text { CFE, } \\
\text { Q. XXXIX-XX }\end{array}$ & $\begin{array}{l}\text { Transbordement er } \\
\text { affluents des transk } \\
\text { jonction. }\end{array}$ \\
\hline Ibid. & CFE, Q. XXXI & $\begin{array}{l}\text { Normes du matérie } \\
\text { faciliter les échang }\end{array}$ \\
\hline 1889 (III) PARIS & M, Q. IX & $\begin{array}{l}\text { Moyens de faire cil } \\
\text { véhicules sur des v } \\
\text { différents (russes e } \\
\text { exemple). }\end{array}$ \\
\hline Ibid. & $\begin{array}{l}\text { CFE, Q. XXVI } \\
\text { Revue d'histoin }\end{array}$ & $\begin{array}{l}\text { Transbordement dt } \\
\text { entre lignes d'écarler, } 26 \text { (printemps 2002) }\end{array}$ \\
\hline
\end{tabular}


(Suite) 
(Suite)

\begin{tabular}{|c|c|c|}
\hline 1900 (VI) PARIS & OG, Q. XXXI & $\begin{array}{l}\text { Clearing houses ol } \\
\text { liquidation. }\end{array}$ \\
\hline lbid. & OG, Q. XXXVI & $\begin{array}{l}\text { Facilitation des vis } \\
\text { réduction des stati } \\
\text { gares frontalières. }\end{array}$ \\
\hline lbid. & $\begin{array}{l}\text { CFE, } \\
\text { Q. XXXVII }\end{array}$ & $\begin{array}{l}\text { Influence des chen } \\
\text { économiques sur li } \\
\text { et sur le trafic des }\end{array}$ \\
\hline $\begin{array}{l}1905 \text { (VII) } \\
\text { WASHINGTON }\end{array}$ & CFE, Q. XVII & $\begin{array}{l}\text { Influence des cher } \\
\text { économiques sur lı } \\
\text { principales. Conco } \\
\text { principaux. }\end{array}$ \\
\hline $\begin{array}{l}1910 \text { (VIII) } \\
\text { BERNE }\end{array}$ & CFE, Q. XX & $\begin{array}{l}\text { Transbordement } d ı \\
\text { entre lignes d'écar }\end{array}$ \\
\hline 1922 (IX) ROME & OG, Q. XIV & $\begin{array}{l}\text { Visite douanière } d \\
\text { internationales de }\end{array}$ \\
\hline Ibid. & OG, Q. XV & $\begin{array}{l}\text { Echange du matéri } \\
\text { indemnités pour re } \\
\text { restitution. }\end{array}$ \\
\hline $\begin{array}{l}1925(X) \\
\text { LONDRES }\end{array}$ & OG, Q. XII & $\begin{array}{l}\text { Gares et lignes col } \\
\text { des dépenses. }\end{array}$ \\
\hline $\begin{array}{l}1930(X \mathrm{I}) \\
\text { MADRID }\end{array}$ & Revue d'histoil & $\begin{array}{l}\text { Néant } \\
\text { e des chemins de te }\end{array}$ \\
\hline
\end{tabular}


(Suite) 